\title{
Indirect measurements of differential gene expression with cDNA microarrays
}

\begin{abstract}
Thomas J. Belbin, John Gaspar, Missak Haigentz, Roman Perez-Soler, Steven M. Keller, Michael B. Prystowsky, Geoffrey Childs, and Nicholas D. Socci Albert Einstein College of Medicine/Montefiore Medical Center, Bronx, NY, USA
\end{abstract}

BioTechniques 36:310-314 (February 2004)

The use of universal RNA reference sets is an increasingly common approach to molecular classification studies with cDNA microarrays. Here we evaluated the reliability of indirect measurements of fluorescence ratios with a common RNA reference as a means of identifying differentially expressed genes. Comparisons of direct and indirect measures of differential gene expression showed a strong overall correlation in fluorescence ratio measurements but also a high degree of false positives in our indirect measurements. These results indicated that the application of more stringent ratio filters may be required when assessing differential gene expression utilizing a common RNA reference in classification studies.

\section{INTRODUCTION}

High-throughput analysis of the expression of thousands of genes in a single experiment has revolutionized the field of molecular biology in recent years. Measurements of differential gene expression using cDNA microarrays rely on the co-hybridization of fluorescently labeled cDNA products prepared independently from the mRNA of two experimental conditions. The ratio of the fluorescence intensities of the incorporated dyes thus represents a ratio of the transcript levels for each of the thousands of genes present on the array. A comparison of this sort allows us to identify genes that alter their expression between two experimental states. While direct comparisons of this type are commonly used in simple microarray hybridization experiments, this approach quickly becomes impractical when trying to compare differential gene expression in a larger population of samples. Under these circumstances, the most popular approach is to cohybridize each sample with a common reference sample and then infer differences in gene expression among the sample population via an indirect ratio measurement (1).

In some studies, such as the comparison of gene expression profiles in a population of tumor samples, the choice of a reference RNA to use is not immediately obvious. One of the more common approaches in use today is a reference pool of RNA produced from a collection of tissues or established cell lines representing a variety of cell types (2). Several features make an RNA sample of this type ideal for use as a common reference. First, the RNA itself is inexpensive and easy to prepare, although the choice of specific sources of RNA to include in the pool can be critical to the success of the study (3). Second, a collection of cell lines represents an inexhaustible source of reference RNA that may be important for long-term studies or those in which the population size is especially large. Third, when a single reference sample is used as a common denominator in several independent studies, the resulting gene expression data can be cross-referenced and compared across all experiments. This approach is now so popular that RNA reference sets of this type are being marketed commercially by several companies [e.g., Stratagene (La Jolla, CA, USA) and BD Biosciences Clontech (Palo Alto, CA, USA)] $(4,5)$.

The purpose of this study was simply to evaluate the accuracy and reliability of differential gene expression hybridization experiments that use a common RNA reference sample and measurements in cDNA microarray indirect measurements of fluorescence ratios. In our experiments, we utilized a cDNA microarray containing 17,841 clones to identify genes differentially expressed between a primary lung adenocarcinoma tissue sample and adjacent healthy lung mucosa from the same patient. We carried out this comparison in two ways. First, we compared gene expression in both samples directly by co-hybridization of corresponding fluorescently labeled cDNAs to a single cDNA microarray. Second, we independently compared gene expression in each sample to a Universal Human Reference (UHR) RNA (Stratagene) using two separate microarray experiments and subsequently inferred expression differences by an indirect method. Both data sets were subsequently compared to an optimum data set derived by two direct measurements of differential gene expression between the two RNA samples in which the fluorescent dyes were reversed.

\section{MATERIALS AND METHODS}

\section{Array Design}

Microarrays were printed using a set of 17,841 sequence-verified human IMAGE cDNA clones representing both known genes and expressed sequence tags (ESTs). These were spotted onto polylysine-coated microscope slides using a custom robot designed and built at the Albert Einstein College of Medicine Microarray Facility (http:// microarray $1 \mathrm{k}$.aecom.yu.edu). Prior to hybridization, these slides were vapormoistened, and the cDNA was fixed onto the slide by cross-linking in a UV Stratalinker ${ }^{\circledR} 2400$ (65 mJ; Stratagene). Following cross-linking, the slides were vapor-moistened a second time, heat-snapped for $4 \mathrm{~s}$ on a hot plate, treated with $0.1 \%$ sodium dodecyl sulfate (SDS) for $20 \mathrm{~s}$, and rinsed with water. In order to completely denature cDNAs present on the array, slides were incubated in water at $95^{\circ} \mathrm{C}$ for 4 min and immediately placed in icecold ethanol. These slides were then centrifuged dry at $1000 \times g$ for $2 \mathrm{~min}$ in an HN-SII clinical centrifuge (International Equipment Company, Needham 
Heights, MA, USA) and used directly for hybridization.

\section{Samples Used, Extract Preparation, and Labeling}

Tissue samples (lung adenocarcinoma and adjacent normal mucosa) were flash-frozen in liquid nitrogen and stored at $-80^{\circ} \mathrm{C}$ prior to RNA extraction. Tissues were homogenized in TRIzoL ${ }^{\circledR}$ reagent using a Model PT 10/35 Tissue Homogenizer (Brinkmann, Westbury, NY, USA) and total RNA was extracted using TRIzOL reagent following the protocol of the manufacturer (Invitro-

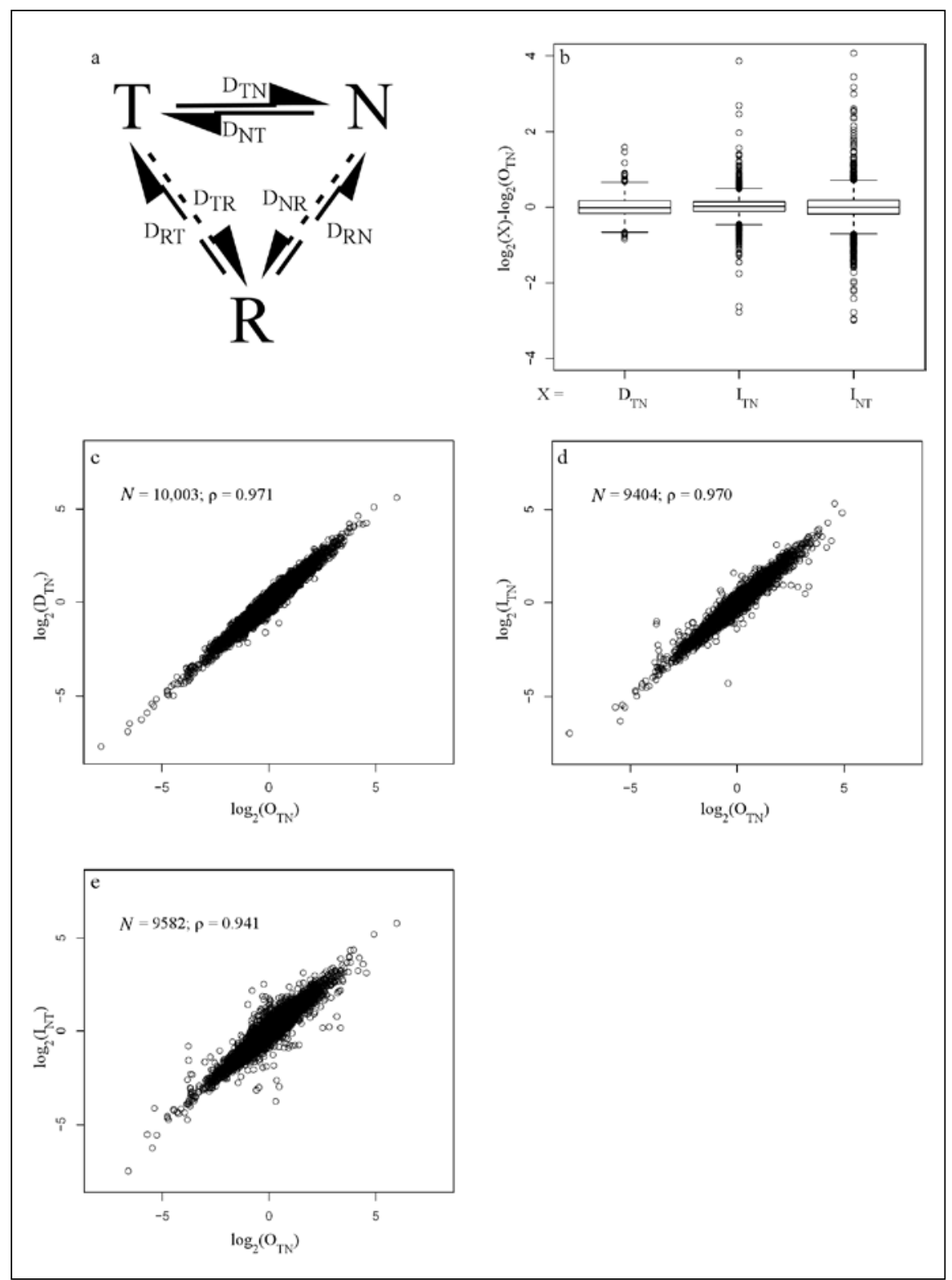

Figure 1. Direct versus indirect measurements of differential gene expression between a lung primary adenocarcinoma (T) and the normal adjacent mucosa (N). (a) Overview of the experimental design. Data sets derived from each comparison are named over each arrow. Indirect data sets $\mathrm{I}_{\mathrm{TN}}$ and $\mathrm{I}_{\mathrm{NT}}$ are indicated by the dotted arrows and dashed arrows, respectively. (b) Box-plots of the ratio of individual direct and indirect data sets to the optimum data set $\mathrm{O}_{\mathrm{TN}}$. Boxes indicate the interquartile range (IQR), defined as the difference between the 25th and 75th percentiles. The line in the middle of each box represents the median ratio. Outliers (values at least two times the IQR from the median) are plotted individually. (c-e) Scatter plots of direct (D) and indirect (I) data set ratio measurements versus the optimum data set $\mathrm{O}_{\mathrm{TN}}$. The number of elements $(N)$ and Pearson correlation coefficient $(\rho)$ are shown in the inset of each plot. 
gen, Carlsbad, CA, USA). Total RNA was subsequently cleaned by column purification using an RNeasy ${ }^{\circledR}$ Mini Total RNA purification kit (Qiagen, Valencia, CA, USA). Quantity and quality of purified RNA was determined by measuring the absorbance at 260 $\mathrm{nm}$ and $280 \mathrm{~nm}$. Aliquots of $5 \mu \mathrm{g}$ total RNA were stored in ethanol at $-80^{\circ} \mathrm{C}$ and used for amplification.

Five micrograms of total RNA from each sample was used to carry out a single round of T7 linear amplification using the MessageAmp ${ }^{\mathrm{TM}}$ T7 Linear Amplification Kit (Ambion, Austin, TX, USA). The quantity and quality of this amplified RNA (aRNA) was determined spectrophotometrically as described above. For each tissue sample, this aRNA represented the pool from which all comparisons involving this sample were used. This was done to avoid the introduction of additional noise into the data set from the T7 linear amplification method. Each aRNA sample was subsequently used in four separate array experiments, two array experiments (dyes reversed) comparing a given sample to each of the other two samples (Figure 1a).

Fluorescent labeling of probes was carried out by reverse transcription of $4 \mu \mathrm{g}$ of amplified RNA with SuperScript ${ }^{\mathrm{TM}}$ II reverse transcriptase (Invitrogen) in a reaction containing $2.5 \mathrm{mM}$ dATP, dCTP, dGTP, 1 mM dTTP, $10 \mathrm{U}$ RNase Out (Invitrogen), $4 \mu \mathrm{L}$ of $\mathrm{Cy}^{\mathrm{TM}}$ labeled dUTP (Amersham Biosciences, Piscataway, NJ, USA) in a final volume of $40 \mu \mathrm{L}$. Reactions were carried out at $42^{\circ} \mathrm{C}$ for $2 \mathrm{~h}$. Fluorescently labeled cDNA probes were combined and purified using a Microcon ${ }^{\circledR}$ YM-50 spin column (Millipore, Billerica, MA, USA).

\section{Hybridization Procedures and Parameters}

Hybridization to cDNA arrays was carried out overnight at $50^{\circ} \mathrm{C}$ in a buffer containing $30 \%$ formamide, $3 \times$ standard saline citrate (SSC), $0.75 \%$ SDS, and $20 \mu \mathrm{g}$ of human Cot-1 DNA. Following hybridization, slides are briefly washed with a solution of $1 \times$ SSC, $0.1 \%$ SDS, then washed for $20 \mathrm{~min}$ at room temperature in $0.2 \times$ SSC, $0.1 \%$ SDS, and $20 \mathrm{~min}$ at room temperature in $0.1 \times \mathrm{SSC}$ (without SDS). Slides are immediately dried by centrifugation and processed using the GenePix ${ }^{\circledR}$ 4000A microarray scanner
(Axon Instruments, Foster City, CA, USA) and GenePix Pro 3.0 software (Axon Instruments) for image processing and subsequent data analysis.

\section{Measurement Data and Specifications}

Red (Cy5) and green (Cy3) signal intensities for each element on the array were calculated using the GenePix Pro 3.0 software. This software gives an integrated intensity per spot for each channel in addition to an integrated background count. In all subsequent analysis, we used the mean background subtracted intensity for the two channels. For each spot, we calculated the mean intensity over the spot in the two channels and from this subtracted the median of the background intensity. We referred to this value simply as the intensity in each channel and denoted them as: $I_{1}$ and $I_{2}$. Before using the spot intensity data for any analysis, it was crucial that the data from each microarray experiment be normalized. We computed an intensity-dependent normalization factor by first finding the rank invariant subset of the spots (the spots that have equal or almost equal
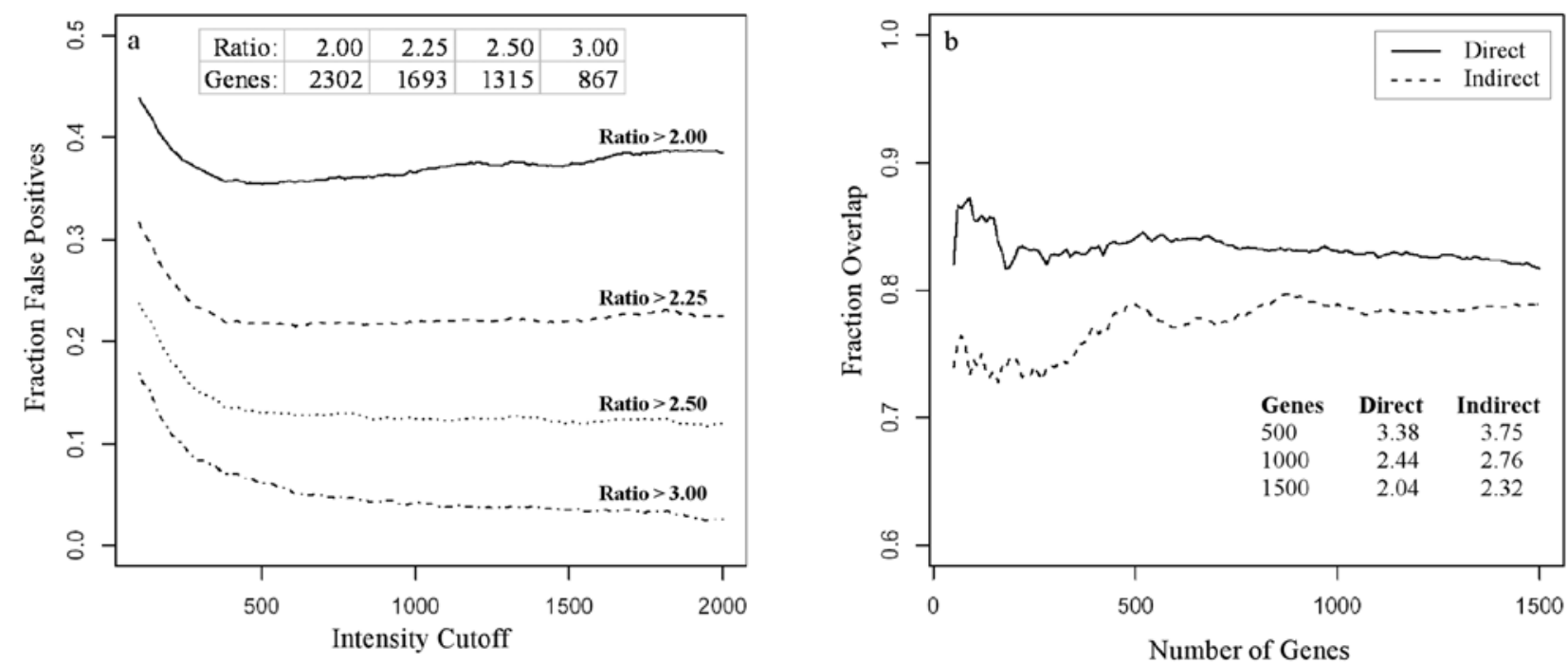

Figure 2. Identification of differentially expressed genes using direct and indirect measurements. (a) Fraction of false positives (genes identified by indirect measurements that are not present in the optimal data set) as a function of average fluorescence intensity cutoff. Table at inset shows the average number of differentially expressed genes identified by the indirect measurement at intensity cutoff of 200, and ratio cutoff measurements of 2.00, 2.25, 2.50, and 3.00. All were compared to the optimum data set of 1575 genes. (b) Fraction of overlap between lists of differentially expressed genes identified by direct (solid line) and indirect (dashed line) measures of gene expression when compared to the optimal list $L(O)$ of differentially expressed genes. Lists of differentially expressed genes from each data set were ranked according to their fluorescence ratio measurement. Fraction of overlap is shown as an average over the range of 50-1500 of the top-ranked genes. Table at inset shows the ratio cutoffs necessary to identify the top 500, 1000, and 1500 genes in direct and indirect measurements of differential gene expression. 
ranks in the two channels) (6). Once this core of spots had been identified, a robust curve was fitted using the lowess function from the $R$ statistical package (7). We then computed the intensitydependent normalization factor $\alpha(A)$ where $A$ was the geometric average intensity $\left(A=\sqrt{I_{1} \times I_{2}}\right)$.

\section{RESULTS AND DISCUSSION}

In order to minimize the complexity of the comparisons in our study, we designed a relatively simple notation to identify the many expression ratios and gene lists that we wanted to compare (Figure 1a). Every measurement of fluorescence ratios $(\mathrm{Cy} 5 / \mathrm{Cy} 3)$ that we examined was derived either from a direct measurement (D) of two samples, or was inferred by an indirect comparison (I) of both samples to the common RNA reference. We defined a set of direct fluorescence ratio measurements $D_{i j}$ as those derived from an experiment in which i was the sample labeled with Cy5 as the fluorescent tag and $\mathrm{j}$ as the sample in which $\mathrm{Cy} 3$ was used. For example, fluorescence ratios belonging to the $\mathrm{D}_{\mathrm{TN}}$ data set represent those obtained from a direct comparison of the tumor and adjacent normal samples in which the tumor RNA (T) was reverselabeled with $\mathrm{Cy} 5$ and the normal tissue RNA (N) with Cy3. We compared tumor RNA to normal adjacent tissue RNA in two direct comparisons, commonly referred to as "dye-flip" array experiments, yielding ratio data sets $\mathrm{D}_{\mathrm{TN}}$ and $\mathrm{D}_{\mathrm{NT}}$. Similarly, we compared each sample RNA directly to the UHR RNA (R), yielding the ratio data sets $\mathrm{D}_{\mathrm{TR}}, \mathrm{D}_{\mathrm{RT}}, \mathrm{D}_{\mathrm{NR}}$, and $\mathrm{D}_{\mathrm{RN}}$. We then defined the indirect measurements of gene expression ratios as follows: we defined the first indirect measurement $\mathrm{I}_{\mathrm{TN}}$ (Figure 1a, dotted arrows) as $\mathrm{I}_{\mathrm{TN}}$ $=\left(\mathrm{D}_{\mathrm{TR}} / \mathrm{D}_{\mathrm{NR}}\right)$, and the second indirect measurement $\mathrm{I}_{\mathrm{NT}}$ (Figure 1a, dashed arrows) as $\mathrm{I}_{\mathrm{NT}}=\left(\mathrm{D}_{\mathrm{RT}} / \mathrm{D}_{\mathrm{RN}}\right)$. Note that for the indirect measurements, we used the same dye (Cy3) in labeling reactions with the reference RNA, as is standard for classification experiments. Therefore, what emerged from our experimental design was four independent data sets $\left(\mathrm{D}_{\mathrm{TN}}, \mathrm{D}_{\mathrm{NT}}, \mathrm{I}_{\mathrm{TN}}\right.$, and $\left.\mathrm{I}_{\mathrm{NT}}\right)$ as measures of differential gene expres- sion between the tumor sample (T) and the adjacent normal tissue $(\mathrm{N})$.

We chose to evaluate the accuracy and reliability of each set of ratio measurements by comparing each to a socalled "optimum" data set $\mathrm{O}_{\mathrm{TN}}$, which we defined as the average of the ratios derived from the two direct measurements $\mathrm{D}_{\mathrm{TN}}$ and $\mathrm{D}_{\mathrm{NT}}$. We examined the distribution of ratio measurements for each direct and indirect measurement by computing the difference in the logarithm of ratios between these measurements and the optimum set for each element on the array (Figure 1b). We observed that the interquartile range (IQR) in single direct ratio data set $\mathrm{D}_{\mathrm{TN}}(-0.167-0.165)$ was comparable to those of the indirect measurements $\mathrm{I}_{\mathrm{TN}}$ $(-0.102-0.137)$ and $\mathrm{I}_{\mathrm{NT}}(-0.178-0.181)$ when each is compared to the optimum ratio measurements. However, the numbers of outliers in both indirect measurements $\left(\mathrm{I}_{\mathrm{TN}}=266, \mathrm{I}_{\mathrm{NT}}=241\right)$ were considerably higher than for the single direct measurement $\left(\mathrm{D}_{\mathrm{TN}}=29\right)$. Scatter plots of ratio measurements showed a high positive correlation when comparing ratio measurements from the optimum set to those derived from direct $\left(\mathrm{D}_{\mathrm{TN}}: N=10,003 ; \rho=\right.$ $0.971)$ and indirect measurements $\left(\mathrm{I}_{\mathrm{TN}}\right.$ : $N=9404 ; \rho=0.970$ and $\mathrm{I}_{\mathrm{NT}}: N=9582$; $\rho=0.941$; where $N$ is the number of elements and $\rho$ is the Pearson correlation coefficient) (Figure 1, b-e).

In addition to correlations between fluorescence ratio measurements, we also examined the actual lists of differentially expressed genes identified in each data set. We first defined a list $L(x)$ of genes determined to be differentially expressed in data set $x$. For the optimum set of differentially expressed genes $L(O)$, a given gene was considered to be differentially expressed if the absolute value of the $\log _{2}$ of its average fluorescence ratio was greater than 1.0 (i.e., its fluorescence ratio was either greater than 2.0 or less than 0.5 ), and its average fluorescence intensity was greater than 300 . This data set was fixed throughout the course of the study. We utilized two approaches to compare other data sets and their corresponding gene lists to the optimum set. First, we examined the degree of false positives in our indirect measurements; that is, the fraction of genes we identified as 
differentially expressed in indirect measurements that were not observed in the optimum set $L(O)$ (Figure 2a). Surprisingly, when utilizing the identical filters for fluorescence ratio and intensity as those used in the optimum set, we found that falsely identified genes made up approximately $35 \%$ $40 \%$ of the population of differentially expressed genes identified by indirect measurements. Furthermore, this number did not decrease significantly when increasing fluorescence intensity cutoffs to as high as 2000 . However, increasing the fluorescence ratio cutoffs had a dramatic effect on the number of false positive in our indirect data sets. An increase from 2.00 to 2.25 in fluorescence ratio cutoff decreased the number of false positives to less than $25 \%$. Increases in the ratio cutoffs to 2.5 decreased the number of false positives to less than $15 \%$ of the optimum set. A ratio cutoff of 3.0 resulted in less than $10 \%$ false positives.

Our second approach was to rank all genes in each list according to the absolute value of its fold-change measurement when comparing tumor tissue $(\mathrm{T})$ and normal adjacent mucosa $(\mathrm{N})$. We then evaluated the fraction of overlap in the lists of differentially expressed genes between a given data set and the optimum data set when taking the top genes from each ranking (Figure 2b). Our results demonstrated that gene lists derived from single direct measurements between tumor and normal RNA samples $\left[L\left(\mathrm{D}_{\mathrm{TN}}\right)\right.$ and $L\left(\mathrm{D}_{\mathrm{NT}}\right)$ ] both showed a $80 \%-85 \%$ overlap with the optimum gene list $L(O)$. Gene lists derived from indirect measurements between tumor and normal samples $\left[L\left(I_{\mathrm{TN}}\right)\right.$ and $\left.L\left(I_{\mathrm{NT}}\right)\right]$ showed a $75 \%-80 \%$ overlap with the optimum gene list $L(O)$ over the range of genes selected.

The reasons for the relatively high number of false positives observed in our indirect measurements are not clear. However, our analysis revealed that one of the causes was the "chip to chip" variation in the measurement of reference RNA (data not shown). Since this variation was additive over two independent measurements, a subset of clones was defined as differentially expressed, not because of differences in the experimental samples being studied, but due to variation in the two reference measurements. Therefore, it is not surprising that an increase in the fluorescence ratio cutoffs would overcome this problem in the data set of indirect measurements and lower the number of false positives. It should also be pointed out that we defined the term "false positive" relative only to our optimal data set. Both the direct and indirect forms of measurement used contain rates of false positives whose determination is outside of the scope of this study. The purpose of this report is to compare bulk data sets directly, with the knowledge that clones of interest identified in a classification study should be confirmed by alternative methods such as real-time PCR or Northern blot analysis.

In conclusion, our results clearly demonstrate that while indirect measurements of differential gene expression using a common RNA reference are often a desirable, and sometimes necessary, approach to classification studies with cDNA microarrays, such indirect measures can generate a significant number of false positives when attempting to identify differentially expressed genes in a population of samples. Surprisingly, this number was consistent over a wide range of signal intensities. However, our results also show that even a modest increase in the ratio cutoffs used to identify differentially expressed genes can dramatically decrease the number of false positive measurements in the population data set. The most obvious drawback of this approach is the discarding of potentially significant data on gene expression differences between samples of interest. Also, it is likely that the magnitude of applied ratio filters would be both laboratory and project-specific. However, it is clear that such initial testing of indirect measurements can dramatically decrease the amount of noise in a classification study that utilizes a common RNA reference.

\section{ACKNOWLEDGMENTS}

The authors wish to thank Dr. Thomas M. Harris, Aldo Massimi, and members of the Albert Einstein College of Medicine (AECOM) Microarray Facility for helpful discussions during the preparation of this manuscript. T.J.B. and J.G. contributed equally to this work.

\section{REFERENCES}

1.Yang, Y.H. and T. Speed. 2002. Design of microarray expression experiments. In $\mathrm{D}$. Bowtell and J. Sambrook (Eds.), DNA Microarrays: A Molecular Cloning Manual. CSH Laboratory Press, Cold Spring Harbor, NY.

2.Puskas, L.G., A. Zvara, L. Hackler, Jr., T. Micsik, and P. van Hummelen. 2002. Production of bulk amounts of universal RNA for DNA microarrays. BioTechniques 33: 898-904.

3.Yang, I.V., E. Chen, J.P. Hasseman, W. Liang, B.C. Frank, S. Wang, V. Sharov, A.I. Saeed, et al. 2002. Within the fold: assessing differential expression measures and reproducibility in microarray assays. Genome Biol. 3:0062.1-0062.12.

4.BD Biosciences Clontech. 2002. Control RNA for microarray experiments. Clontechniques 17:6.

5.Novoradovskaya, N., T. Payette, A. Novoradovsky, J. Braman, N. Chin, A. Pergamenschikov, M. Fero, and D. Botstein. 2000 Pooled high-quality reference RNA for human microarrays. Strategies 13:121-122.

6.Tseng, G.C., M.K. Oh, L. Rohlin, J.C. Liao, and W.H. Wong. 2001. Issues in cDNA microarray analysis: quality filtering, channel normalization, models of variations and assessment of gene effects. Nucleic Acids Res 29:2549-2557.

7.Yang, Y.H., S. Dudoit, P. Luu, D.M. Lin, V. Peng, J. Ngai, and T.P. Speed. 2002. Normalization for cDNA microarray data: a robust composite method addressing single and multiple slide systematic variation. Nucleic Acids Res. 30:e15.

Received 18 September 2003; accepted 10 November 2003.

Address correspondence to Nicholas D. Socci, Departments of Pathology, and the Seaver Center for Computational Biology, Albert Einstein College of Medicine/ Montefiore Medical Center, 1300 Morris Park Avenue, Bronx, NY 10461, USA. e-mail: nsocci@aecom.yu.edu 